\title{
Interpolation of GPS results incorporating geophysical and InSAR information
}

\author{
Linlin Ge, Shaowei Han, and Chris Rizos \\ School of Geomatic Engineering, The University of New South, Sydney, NSW 2052, Australia
}

(Received January 6, 2000; Revised July 25, 2000; Accepted September 2, 2000)

\begin{abstract}
Continuous GPS networks, typically with a station spacing of about $30 \mathrm{~km}$, are still not dense enough to accurately characteristise the dynamics of active faults. Interpolation of these GPS results can improve our understanding of active faults and hence promote related studies. Moreover, even when the networks are densified in order to recover the signature of active faults, the station configuration design may not be ideal. Interpolation at these points, based on the GPS results from a well-designed station network, can provide a good quality control measure. As a first step in the interpolation process an irregular grid pattern is formed, based on the locations of the GPS stations, by using the indexed sorting algorithm. In order to interpolate objectively, the GPS stations and the intended interpolating points are classified into different sub-regions according to their positions in relation to the faults, which are expressed by open- and closed-curve models. GPS results from stations in the same sub-region are used to derive a dynamic model for interpolation at grid points in the same sub-region. A deformation distribution model based on GPS and differential Synthetic Aperture Radar Interferometry (InSAR) results is used as constraints to scale the time series generated using the dynamic model.
\end{abstract}

\section{Introduction}

Large-scale continuous GPS (CGPS) networks for geodynamics have been established over the past decade. However, these networks, the densest of which typically have station spacings of about $30 \mathrm{~km}$, are still not dense enough to accurately characteristise the dynamics of active faults. In fact, if one inspects the resolution requirements for some geophysical and geological applications (as shown in Fig. 1, where the coverage of the current CGPS is indicated by a dashed-line rectangle), the majority of the applications remain unsatisfied. If the rectangle is extended in the negative direction of the vertical axis, this represents a temporal densification of the GPS measurements (Ge et al., 1999). If the rectangle is extended in the negative direction of the horizontal axis, this is a spatial densification of the GPS measurements. This paper will focus on "soft" densification, i.e. spatial densification without deploying more GPS hardware. "Hard" densification, i.e. spatial densification by deploying more GPS receivers (e.g., low-cost, single-frequency receivers) will be discussed in a separate paper (Rizos et al., 1999). Both temporal and spatial densification of GPS measurements is necessary in order to address all the applications.

Interpolation of GPS results (spatial densification especially) can improve our understanding of active faults and hence promote related studies associated with earthquake modeling and hazard mitigation. Moreover, even when the networks are densified in order to recover the signature of active faults, the station configuration design may not be ideal. Interpolation at these points, based on the GPS results from a well-designed station network, can provide a good quality

Copy right $(\mathrm{C})$ The Society of Geomagnetism and Earth, Planetary and Space Sciences (SGEPSS); The Seismological Society of Japan; The Volcanological Society of Japan; The Geodetic Society of Japan; The Japanese Society for Planetary Sciences. control measure.

In order to interpolate objectively both a dynamic model, incorporating geophysical information such as active faults, and a distribution model, incorporating Interferometric Synthetic Aperture Radar (InSAR) information, have to be developed.

\section{Dynamic Model for Interpolation Incorporat- ing Geophysical Information}

As a first step in the interpolation process, an irregular grid pattern is formed based on the locations of the stations in the GPS network. As shown in Fig. 2, the latitudes and longitudes of the GPS stations are read from a data (sequential) file. Here $N_{i j}(i, j=1,2,3$, and 4) refers to the latitude for the $i$ th station in the file and it ranks $j$ among the latitudes for all the GPS stations. $E_{i j}$ stands for longitude and is analogous with $N_{i j}$. Then the latitudes $N_{i j}$ and longitudes $E_{i j}$ are sorted according to their values (i.e. index $j$ ). The sorted latitudes and longitudes are then combined to form the grid $\left(N_{i j}, E_{i j}\right)$, consisting of both existing GPS stations (denoted by " $G$ ") and points to be interpolated (denoted by " $I$ "). The important feature of the two dimensional sorting is that the attributes of GPS stations (index $i$ ) are maintained so that no interpolation operation is needed on grid points of GPS stations. Therefore, the algorithm carries out an "indexed sorting".

In order to interpolate objectively, it is proposed that geophysical information then be incorporated. It is well known that the movements of GPS stations on the two sides of a fault can be significantly different therefore it is important to classify the stations and the intended interpolating points according to their positions in relation to the fault. The open(Fig. 3) and closed- (Fig. 4) curve models have been designed to describe the faults. In the open-curve model as shown in 


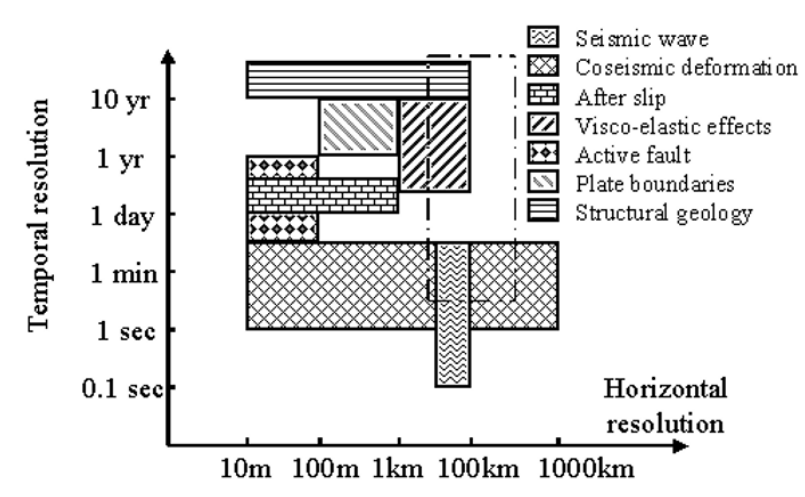

Fig. 1. Resolution requirements of some geophysical and geological applications (various sources).

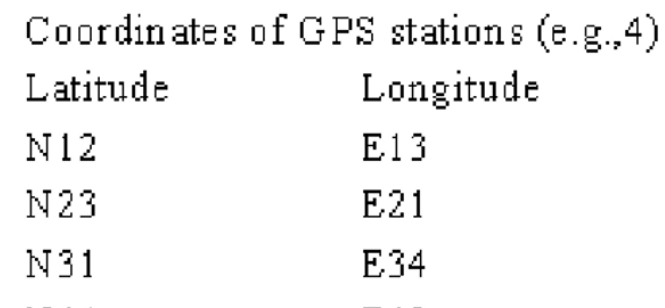

N44

E42

\section{Transformed grid}

\begin{tabular}{|l|l|l|l|l|}
\hline & E21 & E42 & E13 & E34 \\
\hline N 31 & I & I & I & I \\
\hline N 12 & I & I & G & I \\
N 23 & I & I & I & I \\
\hline N 44 & I & G & I & I \\
\hline
\end{tabular}

Fig. 2. An irregular grid formed by indexed sorting.

Fig. 3, the region of interest is divided into upper (or left) and lower (or right) sub-regions by an open curve. This model should be adequate for most seismic faults.

An algorithm has been developed to automatically identify in which sub-region a station or an interpolating point belongs to. Assume the unit vectors of latitude is $\vec{i}$ and longitude is $\vec{j}$, from an arbitrary point on the fault $\left(N_{i}, E_{i}\right)$ to the GPS station (GS) at $\left(N_{G}, E_{G}\right)$ the vector can be expressed as:

$$
\vec{G}=\left(N_{G}-N_{i}\right) \cdot \vec{i}+\left(E_{G}-E_{i}\right) \cdot \vec{j} .
$$

A minimum search of vector length is performed to find $|\vec{G}|_{\left(N_{i}, E_{i}\right)}=\min$.

Therefore, a GS local fault vector can be written as:

$$
\vec{F}_{G}=\left(N_{i+1}-N_{i}\right) \cdot \vec{i}+\left(E_{i+1}-E_{i}\right) \cdot \vec{j} .
$$

From an arbitrary point on the fault $\left(N_{j}, E_{j}\right)$ to the interpolating point (IP) at $\left(N_{I}, E_{I}\right)$ the vector can be expressed as:

$$
\vec{I}=\left(N_{I}-N_{j}\right) \cdot \vec{i}+\left(E_{I}-E_{j}\right) \cdot \vec{j} .
$$

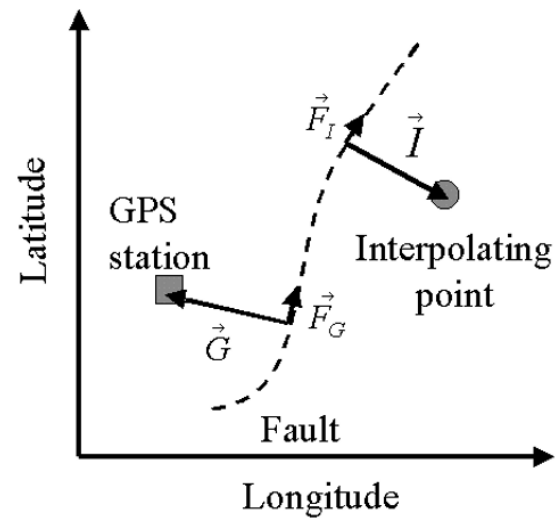

Fig. 3. Open-curve model: one GPS station and one interpolating point case.

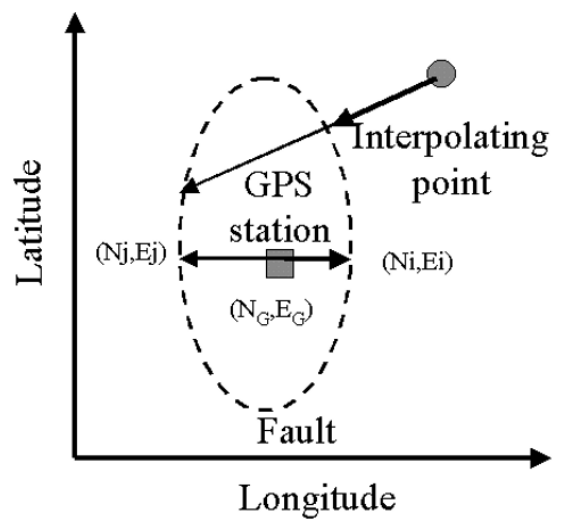

Fig. 4. Closed-curve model: one GPS station and one interpolating point case.

A minimum search of vector length is performed to find $|\vec{I}|_{\left(N_{j}, E_{j}\right)}=\min$.

Then, an IP local fault vector can be written as:

$$
\vec{F}_{I}=\left(N_{j+1}-N_{j}\right) \cdot \vec{i}+\left(E_{j+1}-E_{j}\right) \cdot \vec{j} .
$$

From Eqs. (1) to (4) a "decision" can be made: if $\left(\vec{F}_{G} \times \vec{G}\right)$. $\left(\vec{F}_{I} \times \vec{I}\right)>0$ the GPS station and the interpolating point are on the same side of the fault. Otherwise they are on different sides of the fault.

In the closed-curve model as shown in Fig. 4, the region to be studied is divided into outside and inside sub-regions by a closed curve. This model is appropriate for applications such as volcano deformation monitoring.

A different algorithm for this model is developed to automatically identify in which sub-region a station or interpolating point belongs to. Again assume the unit vectors of latitude is $\vec{i}$ and longitude is $\vec{j}$. From the GPS station (GS) at $\left(N_{G}, E_{G}\right)$ to an arbitrary point on the fault $\left(N_{i}, E_{i}\right)$ the vector can be expressed as:

$$
\vec{G}=\left(N_{i}-N_{G}\right) \cdot \vec{i}+\left(E_{i}-E_{G}\right) \cdot \vec{j} .
$$

A minimum search of vector length is performed to find $|\vec{G}|_{\left(N_{i}, E_{i}\right)}=\min$. 
The GS primary vector is thus determined as:

$$
\vec{L}_{1}=\vec{G}
$$

Then a minimum distance search from an arbitrary point on the fault to the GS primary vector can determine $\left(N_{j}, E_{j}\right)$ which is used to calculate the GS secondary vector:

$$
\vec{L}_{2}=\left(N_{j}-N_{G}\right) \cdot \vec{i}+\left(E_{j}-E_{G}\right) \cdot \vec{j}
$$

From Eqs. (5) and (6), a "decision" can be made: if $\vec{L}_{1} \cdot \vec{L}_{2}>$ 0 the GPS station is outside of the fault. Otherwise it is inside the fault. The position of the interpolating point relative to the fault can be determined in the same way.

The combination of open- and closed-curve models can deal with comparatively complex fault systems. After the classification, GPS results from stations in the same subregion are used to derive a dynamic model, which is used to interpolate at grid points in the same sub-region. Figure 5 is an example of a dynamic model extracted using adaptive filtering $(\mathrm{Ge}, 1999)$ on CGPS results from two closely located stations in the same classified group: stations BRAN and LEEP of the Southern California Integrated GPS Network (SCIGN). The first two plots are latitude time series for stations BRAN and LEEP respectively. The third plot is the model derived using an adaptive filter, which can be used as a dynamic model for interpolation in temporal domain. The last plot in Fig. 5 is the local monument movement at BRAN output by the filter. It is well known that the local monument movement in a continuous GPS station can be very much misleading. Although much effort has been made to improve the design of the monument, this local movement remains a significant concern in the interpretation. If the GPS time series from indivisual CGPS stations as shown in the first two plots of Fig. 5 are used directly as dynamic model for interpolation, all the deformations at grid points around the stations will be biased. Therefore, it is important to separate the local monument movement from the crustal deformation due to plate or fault movements. Fortunately,

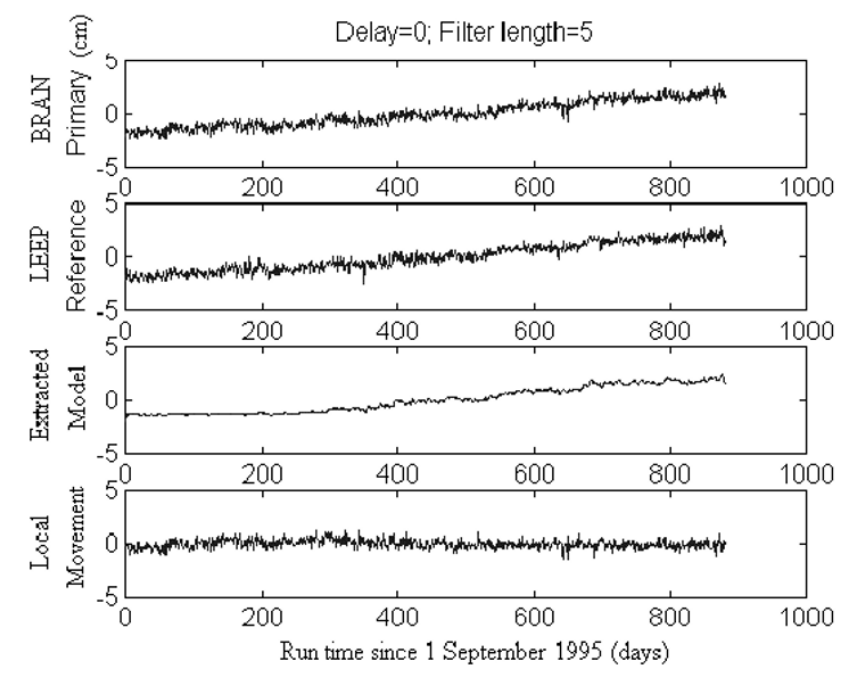

Fig. 5. Dynamic model extracted using adaptive filtering on CGPS results from BRAN and LEEP stations of SCIGN (latitude component, data courtesy of JPL). since the local monument movements are independent while the crustal deformations at stations in the same sub-region are strongly correlated as evident in Fig. 5, the adaptive filtering can be employed to decompose them. Even though the two time series from BRAN and LEEP are very different when observed carefully, the derived model in the third plot can function reliably as the dynamic model.

\section{Distribution Model for Interpolation Incorpo- rating InSAR Information}

Interferometric Synthetic Aperture Radar (InSAR) is a technique first suggested in 1974 (Graham, 1974). After developments over more than two decades, InSAR is now capable of addressing many applications such as mapping topography, detecting topographic change, etc. InSAR has approximately $25 \mathrm{~m}$ spatial resolution. Without the need for any ground-based receiver or cooperative target, it can virtually monitor every corner of the earth. But InSAR is very sensitive to errors such as due to atmospheric effects (tropospheric delay, ionospheric delay, etc.), satellite orbit error, conditions of the ground surface and temporal decorrelation. When presented in the InSAR image, these errors can lead to misleading interpretation. There is at present no way to eliminate them using SAR data alone.

Therefore, a distribution model for interpolation based on both GPS and InSAR results is proposed, as illustrated in Table 1. In some sites of interest there are both GPS and InSAR results for the deformation. But in most other sites there will only be InSAR-derived results. All of the results are input into a least-square adjustment. The adjusted results are used as constraints to scale the time series generated at the interpolated points based on the dynamic model. It is important to have overlapping GPS and InSAR results over the active fault region so that the GPS results can be used to calibrate out some errors in the InSAR results. But this condition is very hard to satisfy (Bock and Williams, 1997). It is hoped that the densification of CGPS and new missions for InSAR (JPL, 1999), will ease such difficulties.

Figure 6 is an example of the deformation distribution model based on both InSAR and GPS for the 1992 Landers Earthquake following such an approach. The geocoded InSAR result and GPS result of 38 sites for the 1992 Landers Earthquake epicenter region were kindly provided by courtesy of Dr. Massonnet of CNES, France (Massonnet et al., 1993) and Dr. Freymueller of the University of Alaska, US

Table 1. Distribution model incorporating InSAR information.

\begin{tabular}{cccc}
\hline $\begin{array}{c}\text { Interested } \\
\text { site }\end{array}$ & $\begin{array}{c}\text { GPS } \\
\text { result }\end{array}$ & $\begin{array}{c}\text { InSAR } \\
\text { result }\end{array}$ & $\begin{array}{c}\text { Least-square } \\
\text { adjusted result }\end{array}$ \\
\hline 1 & G1 & S1 & A1 \\
2 & G2 & S2 & A2 \\
3 & G3 & S3 & A3 \\
4 & G4 & S4 & A4 \\
5 & & S5 & A5 \\
6 & & S6 & A6 \\
\hline
\end{tabular}




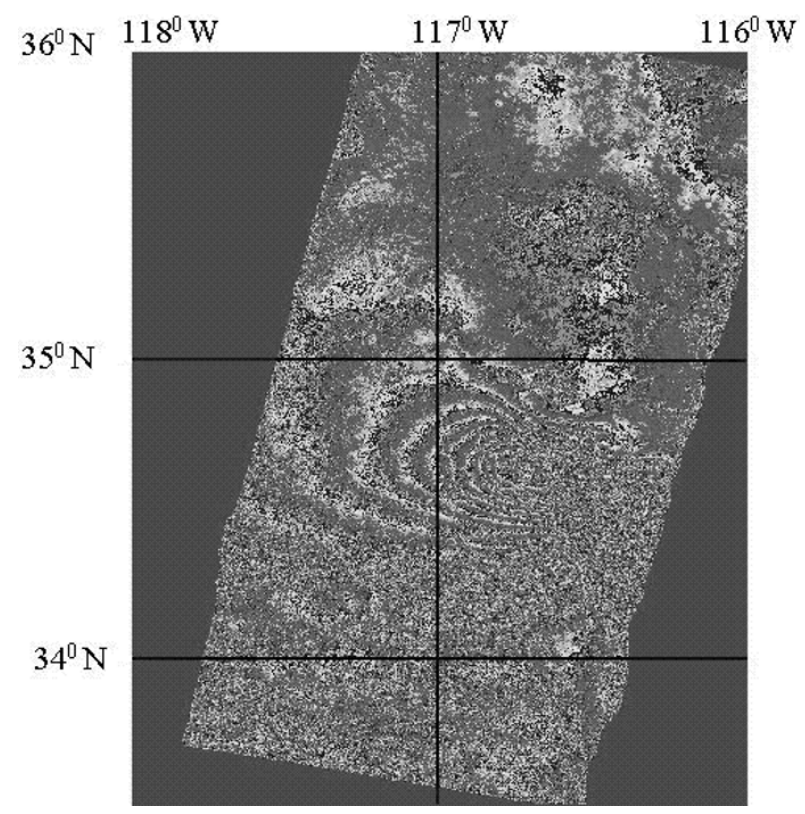

Fig. 6. A deformation distribution model based on both InSAR and GPS for the 1992 Landers Earthquake.

(Freymueller et al., 1994) respectively. In order to derive the distribution model for interpolation in spatial domain, GPS sites were first matched with their imaging points in the InSAR result. Then the GPS displacements were projected to the change in range in the direction which points toward the satellite. Meanwhile, the InSAR result was resampled. Having obtained both InSAR and GPS results, they were least-square adjusted as outlined above. Figure 6 is a plot of the adjusted result, which is not significantly different from the InSAR alone result (Massonnet et al., 1993) due to the facts that some GPS stations have been used as ground control points in the InSAR data processing and that the InSAR and GPS results employed are generally in good agreement. However, this will in no means undermining the importance of InSAR/GPS integration in deriving the distribution model.

\section{Concluding Remarks}

Geophysical and InSAR information have been incorporated in the interpolation of GPS measurements. The indexed sorting algorithm is very effective in forming an irregular grid pattern for interpolation and maintaining the "attributes" of the GPS stations. The combination of open- and closed-curve models of a fault is suitable for dealing with comparatively complex fault systems. Adaptive filtering has been successfully used to extract a dynamic model from the GPS time series of stations in the same classified group. A deformation distribution model for interpolation in spatial domain was derived based on both InSAR and GPS results for the 1992 Landers Earthquake.

The work described in this paper is part of a larger study on the use of the Double Interpolation and Double Prediction (DIDP) scheme to integrate GPS and InSAR results.

Acknowledgments. The first author is sponsored by an International Postgraduate Scholarship of the Australian Government. This study is partly supported by a grant from the Australian Research Council (ARC). Dr. Satoshi Fujiwara of the Geographical Survey Institute of Japan and an anonymous reviewer are acknowledged for useful comments for the authors to improve the manuscript.

\section{References}

Bock, Y. and S. Williams, Integrated satellite interferometry in Southern California, EOS Trans., $A G U, \mathbf{7 8}(29), 293,1997$.

Freymueller, J. T., N. E. King, and P. Segall, The co-seismic slip distribution of the Landers earthquake, Bull. Seis. Soc. America, 84(3), 646-659, 1994.

Ge, L., GPS seismometer and its signal extraction, Proc. 12th Int. Tech. Meeting of the Satellite Division of the U.S. Inst. of Navigation GPS ION'99, Nashville, Tennessee, 14-17 September, 1999.

Ge, L., S. Han, C. Rizos, Y. Ishikawa, M. Hoshiba, Y. Yoshida, M. Izawa, N Hashimoto, and S. Himori, GPS seismometers with up to $20 \mathrm{~Hz}$ sampling rate, Pres. International Symposium on GPS-Application to Earth Sciences and Interaction with Other Space Geodetic Techniques (Tsukuba GPS 99), Tsukuba, Japan, 18-22 October, 1999.

Graham, L. C., Synthetic interferometer radar for topographic mapping, Proc. IEEE, 62, 763-768, 1974.

JPL, http: //www.jpl.nasa.gov/missions/future/, 1999.

Massonnet, D., M. Rossi, C. Carmona, F. Adragna, G. Peltzer, K. Feigl, and T. Rabaute, The displacement field of the landers earthquake mapped by radar interferometry, Nature, 364(6433), 138-142, 1993.

Rizos, C., S. Han, L. Ge, H. Y. Chen, Y. Hatanaka, and K. Abe, Low-cost densification of permanent GPS networks for natural hazard mitigation: first tests on GSI's GEONET network, Pres. Tsukuba GPS 99, Tsukuba, Japan, 18-22 October, 1999.

L. Ge (e-mail: 1.ge@student.unsw.edu.au), S. Han, and C. Rizos 